\title{
Determinação do módulo de elasticidade da madeira em função da inclinação das fibras utilizando tomógrafo acústico
}

\author{
Determination of modulus of elasticity of wood \\ depending on the inclination of fibers using \\ acoustic tomography
}

\author{
Edgar Vladimiro Mantilla Carrasco ${ }^{1}$, Marina de Faria Souza ${ }^{2}$, \\ Lívia Ramos Santos Pereira ${ }^{3}$, Caio Brant Vargas ${ }^{4}$, \\ Judy Norka Rodo Mantilla ${ }^{5}$
}

\footnotetext{
${ }^{1}$ Escola de Arquitetura - TAU/UFMG, Belo Horizonte, MG. e-mail: mantilla@dees.ufmg.br

${ }^{2}$ Escola de Arquitetura - TAU/UFMG, Belo Horizonte, MG. e-mail: marinafaria25@gmail.com

${ }^{3}$ Escola de Engenharia - DEES/UFMG, Belo Horizonte, MG. e-mail: liviarsp.eng@gmail.com

${ }^{4}$ Escola de Arquitetura - TAU/UFMG, Belo Horizonte, MG. e-mail: caiobranstvargas@gmail.com

${ }^{5}$ Faculdade de Engenharia e Arquitetura - Universidade FUMEC, Belo Horizonte, MG. e-mail: judy.mantilla@fumec.br
}

\section{RESUMO}

Os objetivos deste trabalho são determinar, a partir de ensaios não destrutivos, o módulo de elasticidade em função da inclinação das fibras da madeira através de um tomógrafo acústico, comparar e discutir a aplicação de seis critérios de falha adaptados para ensaios não destrutivos e modificar as equações para determinar os critérios de falha mais adequados para o ensaio não destrutivo proposto. Para isto foram ensaiados 6 corpos de prova de 150x15x4 $\mathrm{cm}$ de 6 espécies de madeira. Utilizou-se um tomógrafo acústico com oito transdutores. Estes sensores são fixados e excitados mecanicamente com um martelo. Cada vez que um sensor é excitado os outros captam o tempo que a onda mecânica tardou em chegar, logo é determinada a velocidade e calculado o módulo de elasticidade. A inclinação das fibras é determinada a partir dos ângulos formados por cada transdutor em relação a direção destas. Os resultados experimentais foram comparados com seis teorias de critérios de ruptura mecânica. Conclui-se que é possível utilizar o tomógrafo acústico para determinar o módulo de elasticidade inclinado ás fibras, com apenas um ensaio experimental. Todos os seis modelos matemáticos avaliados não apresentam significância estatística em seu formato original. Com as modificações feitas nos modelos os que apresentaram significância foram apenas os modelos: hiperbólica, Karlsen e Hankinson, portanto eles são os mais indicados para estimar os valores do módulo de elasticidade dinâmico em função da inclinação das fibras.

Palavras-chave: madeira; módulo de elasticidade; ensaios não destrutivos; tomógrafo acústico.

\section{ABSTRACT}

The objectives of this work are to determine, from non-destructive tests, the modulus of elasticity as a function of the inclination of the wood fibers through an acoustic tomograph, to compare and discuss the application of six fault criteria adapted for non destructive tests and to modify the formulas for to determine the most suitable failure criteria for the proposed non-destructive test. To determine the most suitable failure criteria for the proposed non-destructive test. For this, 6 specimens of 150x15x4 cm of 6 species of wood were tested. An acoustic tomograph with eight transducers was used. These sensors are fixed and mechanically excited with a hammer. Each time a sensor is excited the others capture the time the mechanical wave took to arrive, then the speed is determined and the modulus of elasticity is calculated. The inclination of the fibers is determined from the angles formed by each transducer in relation to the direction thereof. The experimental results were compared with six theories of mechanical rupture criteria. It is concluded that it is possible to use the acoustic tomograph to determine the modulus of elasticity inclined to the fibers, with only one experimental test. All six mathematical models evaluated do not present statistical significance in their original format. With the modifications made in the models those that presented significance were only the models: 
hyperbolic, Karlsen and Hankinson, therefore they are the most indicated to estimate the values of the modulus of elasticity as a function of the inclination of the fibers.

Keywords: wood, modulus of elasticity, nondestructive testing, acoustic tomography.

\section{INTRODUÇÃO}

A maioria dos estudos usando o método de propagação de ondas está associada com ultrassons [1]. No entanto, alguns trabalhos [2-7] utilizaram o princípio da propagação das ondas de tensão para estimar as propriedades da madeira.

O uso de ondas acústicas com frequências maiores a $20 \mathrm{kHz}$ são características dos ensaios não destrutivos que utilizam os métodos de ultrassom, e quando, os corpos de prova, são excitados com choques de martelo são emitidas ondas mecânicas. Utilizando o método de propagação das ondas de tensão através da tomografia acústica (TA) é possível determinar com maior precisão as características da madeira, uma vez que o tomógrafo fornece uma gama de velocidades e não apenas um único valor de velocidade, como faz o ultrassom.

Alguns autores [2, 6, 8-11] utilizaram a técnica de ondas de tensão com o único objetivo de obter uma imagem tomográfica da madeira. No trabalho [12] os autores utilizaram o Tomógrafo Acústico para determinar o módulo de elasticidade normal às fibras. A determinação do módulo de elasticidade em função da inclinação das fibras da madeira utilizando o TA ainda não foi realizado.

O comportamento da madeira à compressão quando solicitada inclinada às fibras já é um fato conhecido $[13,14,15]$ e a maior ou menor influência, do módulo de elasticidade, depende da espécie da madeira. Quanto à influência da inclinação das fibras no módulo de elasticidade dinâmico, não se encontrou estudos que definam seu comportamento. Trabalhos a respeito do comportamento de ligações solicitadas ao cisalhamento em função da inclinação das fibras são apresentados pelos autores [16, 17,18]. Estes autores também apresentam diversas formulações levando em consideração as teorias de falhas.

A madeira é um material único e variável devido a seus defeitos naturais e características anisotrópicas. Assim as teorias de falha são muito complexas, $[19,20]$. Não se tem disponíveis na literatura critérios de falha para determinar o módulo de elasticidade dinâmico em função da inclinação das fibras da madeira. Apenas existem critérios de falha para compressão, tração e cisalhamento da madeira. Entre os numerosos critérios de falha, as equações de Hankinson, Karlsen, DIN-1052, Keylwerth, Hiperbólica e Teoria de TsaiHill, são as mais utilizadas para estimar a características mecânicas da madeira quando solicitada inclinada às fibras.

Neste sentido os objetivos deste estudo são: determinar, a partir de ensaios não destrutivos, o módulo de elasticidade dinâmico em função da inclinação das fibras da madeira utilizando o tomógrafo acústico, comparar e discutir a aplicação de seis critérios de falha adaptados para ensaios não destrutivos, e, modificação das diversas equações visando determinar os critérios de falha adequados para o ensaio não destrutivo proposto.

\section{MATERIAIS E MÉTODOS}

Para os ensaios não destrutivos foram utilizadas 6 vigas de 6 espécies de madeira, num total de 36 vigas, que serão chamados de corpos de prova (CPs). As dimensões das vigas eram de 150x15x4cm (CxLxE) e as espécies foram: Angelim [Hymenolobium petraeum], Parajú [Manilkara ssp], Cumarú [Dipteryx odorata]. Pinus eliotte [Pinus elliottii Engelm.], Sucupira [Diplotropis ssp] e Eucaplito grandis [Eucalyptus grandis], Figura 1. Todas as vigas foram condicionadas em ambientes com temperatura e umidade controlada por período adequado até que todas as madeiras estejam com umidade estabilizada de $12 \%$, sendo que as variações foram de $0,15 \%$.

Todos os ensaios foram realizados no Centro de Pesquisa Avançado da Madeira e novos Materiais (CPAM3), da Universidade Federal de Minas Gerais (UFMG).

A densidade dos CPs foi determinada utilizando uma balança com sensibilidade de $0,01 \mathrm{~g}$ e capacidade de $5 \mathrm{~kg}$, um paquímetro com sensibilidade de $0,01 \mathrm{~mm}$ e uma trena de $5 \mathrm{~m}$. A umidade e a densidade aparente foram determinadas de acordo com a norma brasileira NBR 7190 [21].

Para os ensaios não destrutivos foi utilizado o Tomógrafo Acústico (TA) da Fakopp 3D, com 8 transdutores piezos elétricos, os quais foram inseridos simétrica e ordenadamente no CP. Antes do início dos ensaios, marcou-se os corpos de prova com traços nas laterais, para indicar os locais dos transdutores. Em cada peça e em um plano horizontal, foram acoplados oito sensores sendo 2 sensores em cada aresta. Estes sensores são fixados com o uso de um martelo de borracha. 
Na Figura 2.a visualiza-se a posição dos transdutores instalados e prontos para o início do ensaio de AT. Este ensaio consiste em excitar cada transdutor com pancadas através de um martelo metálico, emitindo assim uma onda mecânica. Um computador foi utilizado para monitoramento por meio do software ArborSonic, no qual são inseridas informações sobre a família da madeira, as dimensões do CP, a disposição e a localização cartesiana dos sensores. O software fornece o tempo de percurso do pulso entre o sensor excitado e os outros sensores, posicionados em diversos ângulos em relação à orientação das fibras da madeira, e calcula a velocidade de cada onda mecânica, fornecendo uma matriz de velocidades com 42 elementos.

Com o auxílio do Software AutoCAD foram desenvolvidos modelos digitais de alta precisão dos corpos de prova, permitindo a análise dos ângulos entre os transdutores e a relação destes ângulos com a inclinação das fibras.

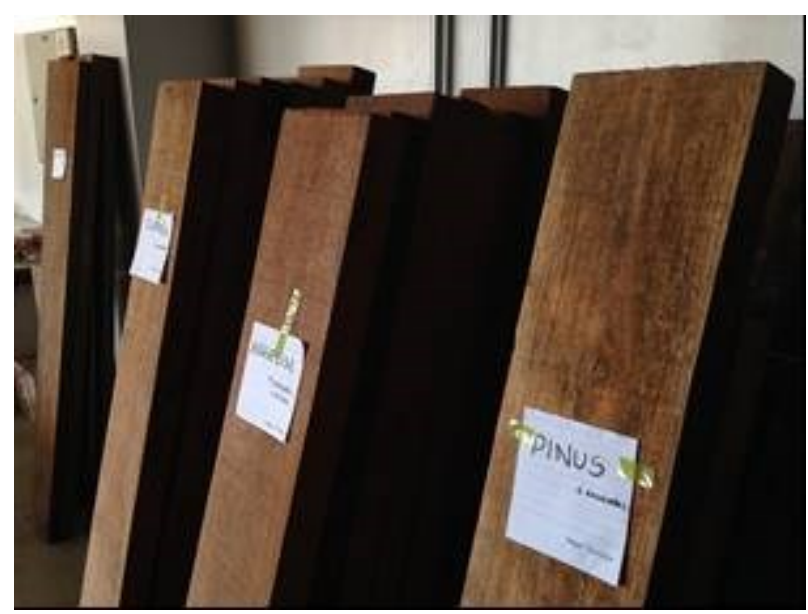

a)

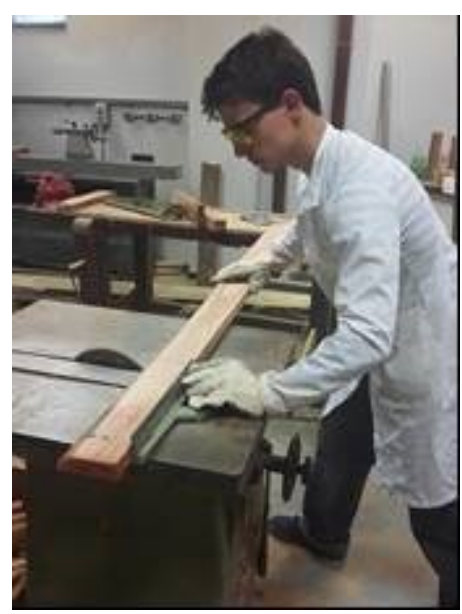

b)

Figura 1: a) CP utilizados para ensaios, b) Corte e adequação das dimensões dos CP.

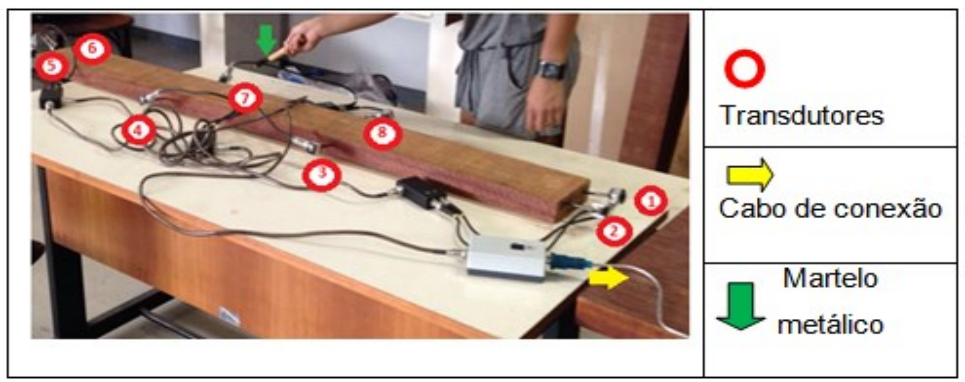

a)

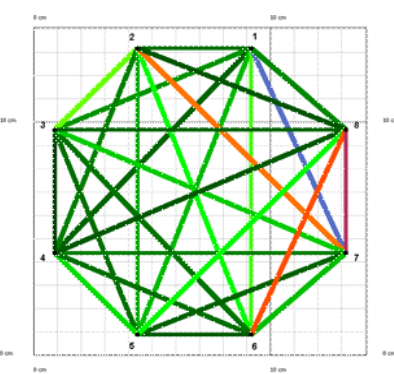

(b)

Figura 2: a) Ensaio com o tomografo, b) Imagem isocromática da velocidade de cada onda mecânica.

\subsection{Fundamentação teórica}

As seis equações apresentadas a seguir são decorrentes da aplicação de diversos critérios de falha, utilizados para obter a resistência à compressão inclinada às fibras. Estas equações serão modificadas para o módulo de elasticidade dinâmico $\left(\mathrm{E}_{\mathrm{d}}\right)$ normalizado em função da inclinação das fibras (módulo de elasticidade dinâmico inclinado/módulo de elasticidade dinâmico paralelo as fibras $\left.=\mathrm{E}_{\mathrm{d} \theta} / \mathrm{E}_{\mathrm{d} 0}\right)$.

Para Kollmann e Côté [13] o modelo empírico proposto por Hankinson, equação 1, com expoente n=2, poderá ser utilizado também para determinar o E inclinado às fibras. Já [22] recomendam o modelo empírico apresentado na equação 2, com expoente n=3. A norma alemã DIN 1052 [23] utiliza a equação 3 com o expoente $n=1$. Segundo [13], Keylwerth também desenvolveu uma expressão empírica com expoente $n=2$, mas para a variação do E em função da inclinação das fibras, equação 4. 


$$
\begin{aligned}
& \frac{E_{d \theta}}{E_{d 0}}=\frac{\frac{E_{d 0}}{E_{d 0}} \times \frac{E_{d 90}}{E_{d 0}}}{\frac{E_{d 0}}{E_{d 0}} \times \sin (\theta)^{\mathrm{n}}+\frac{E_{d \theta}}{E_{d 0}} \times \cos (\theta)^{\mathrm{n}}} \\
& \frac{E_{d \theta}}{E_{d 0}}=\frac{\frac{E_{d 0}}{E_{d 0}}}{1+\left(\frac{\frac{E_{d 0}}{E_{d 0}}}{\frac{E_{d 90}}{E_{d 0}}}+1\right) \times \sin (\theta)^{\mathrm{n}}} \\
& \frac{E_{d \theta}}{E_{d 0}}=\frac{E_{d 0}}{E_{d 0}}-\left(\frac{E_{d 0}}{E_{d 0}}-\frac{E_{d 90}}{E_{d 0}}\right) \times \sin (\theta)^{\mathrm{n}}
\end{aligned}
$$

Woodward e Minor [19] apresentam a teoria de Tsai-Hill como sendo uma extensão do critério de Von Mises para materiais isotrópicos. Para o caso de ensaios com carregamento em uma direção tem a forma da equação 5, com expoente $n=2$. Ainda [24] afirma que a teoria de Tsai-Hill e a equação de Hankinson superestimam a resistência para pequenas inclinações das fibras da madeira e propõe uma fórmula hiperbólica, equação 6 , com o valor de n=0,01. Há controvérsia, entre os autores, sobre o valor do expoente (n) dos termos trigonométricos nas equações 1 a 6 . Alguns autores indicam coeficientes diferentes conforme o tipo de solicitação, outros indicam coeficientes diferentes para diferentes teores de umidade da madeira. Este último comentário também foi comprovado por $[16,17,18]$.

$$
\begin{aligned}
& \frac{E_{d \theta}}{E_{d 0}}=\frac{\frac{E_{d 0}}{E_{d 0}}}{\left(\cos (\theta)^{\mathrm{n}}-\frac{\frac{E_{d 0}}{E_{d 0}}}{\frac{E_{d 90}}{E_{d 0}}} \times \sin (\theta)^{\mathrm{n}}\right) \times \cos (2 \theta)+\frac{\frac{E_{d 0}}{E_{d 0}}}{\frac{E_{d 45}}{E_{d 0}}} \times \sin (2 \theta)^{\mathrm{n}}} \\
& \frac{1}{\frac{E_{d \theta}{ }^{2}}{E_{d 0}}}=\frac{\frac{\cos (\theta)^{2 \mathrm{n}}}{\frac{E_{d 0}{ }^{2}}{E_{d 0}}}-\frac{\cos (\theta)^{\mathrm{n}} \sin (\theta)^{\mathrm{n}}}{\frac{E_{d 0}{ }^{2}}{E_{d 0}}}+\frac{\sin (\theta)^{2 \mathrm{n}}}{\frac{E_{d 90}{ }^{2}}{E_{d 0}}}}{\frac{E_{d 0}}{E_{d 0}} \times \frac{E_{d 90}}{E_{d 0}}} \quad \frac{E_{d \theta}}{E_{d 0}}=\frac{2 \frac{E_{d 0}}{E_{d 0}} \times \frac{E_{d 90}}{E_{d 0}}}{\mathrm{e}^{\mathrm{n} \theta\left(\frac{E_{d 0}}{E_{d 0}}+\frac{E_{d 90}}{E_{d 0}}\right)+\mathrm{e}^{-\mathrm{n} \theta\left(\frac{E_{d 90}}{E_{d 0}}-\frac{E_{d 0}}{E_{d 0}}\right)}}}
\end{aligned}
$$

\section{RESULTADOS E DISCUSSÕES}

Com as velocidades (v) e a densidade do corpo de prova $(\rho)$ foi possível calcular o módulo de elasticidade dinâmico, a partir da equação 7 :

$$
\mathrm{E}_{d}=\frac{\mathrm{v}^{2}}{\rho}
$$

Os ângulos entre a emissão da onda e a direção das fibras foram determinados com auxílio do software AutoCad. Na Figura 3 cada imagem representando um corpo de prova com suas dimensões e os pontos nos quais situavam-se os transdutores. Adotou-se como hipótese que as fibras da madeira estavam dispostas longitudinalmente nas vigas ensaiadas, sendo os ângulos calculados a partir da orientação da fibra até as linhas de propagação das ondas. 


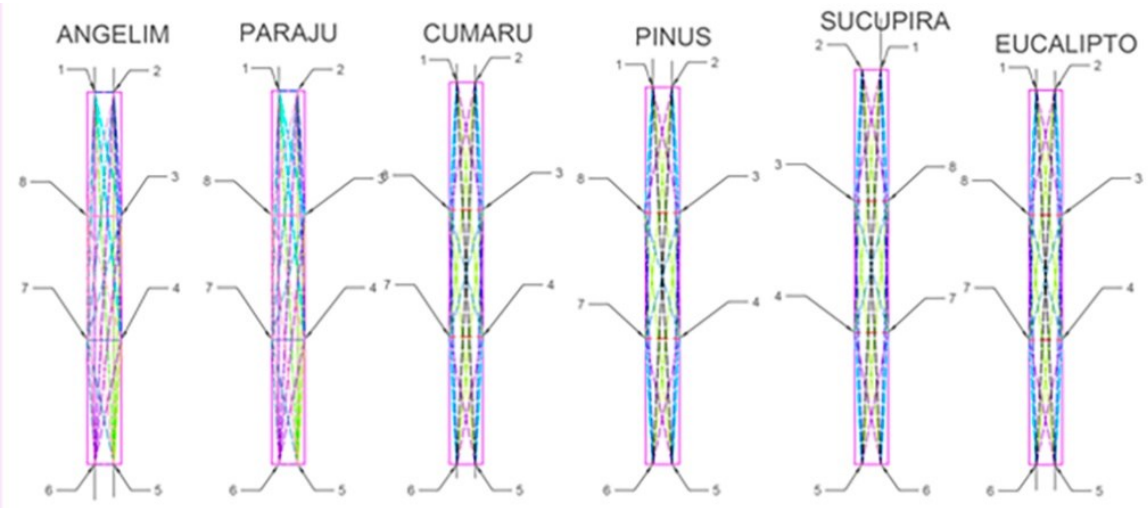

Figura 3: Esquema de trajetória das ondas sonoras nos CP e respectivos ângulos entre os transdutores.

Assim, para cada corpo de prova ensaiado, obteve-se uma matriz quadrada $8 \times 8$ e simétrica (Figura 4). Aplicando-se a equação 7 obtém-se os módulos de elasticidade dinâmicos em função da inclinação das fibras.

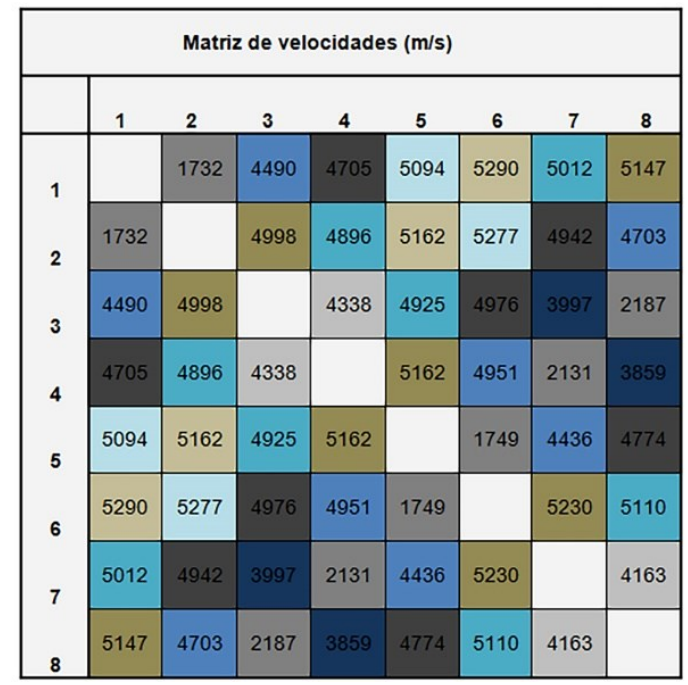

Figura 4: Matriz de velocidades obtidas para um CP.

Na Figura 5 é mostrado um gráfico contendo todos os resultados dos ensaios de todas as espécies de madeira. No eixo vertical tem-se a relação do módulo de elasticidade inclinado às fibras/módulo de elasticidade paralelo às fibras $\left(\mathrm{E}_{\mathrm{d} \theta} / \mathrm{E}_{\mathrm{d} 0}\right)$. 


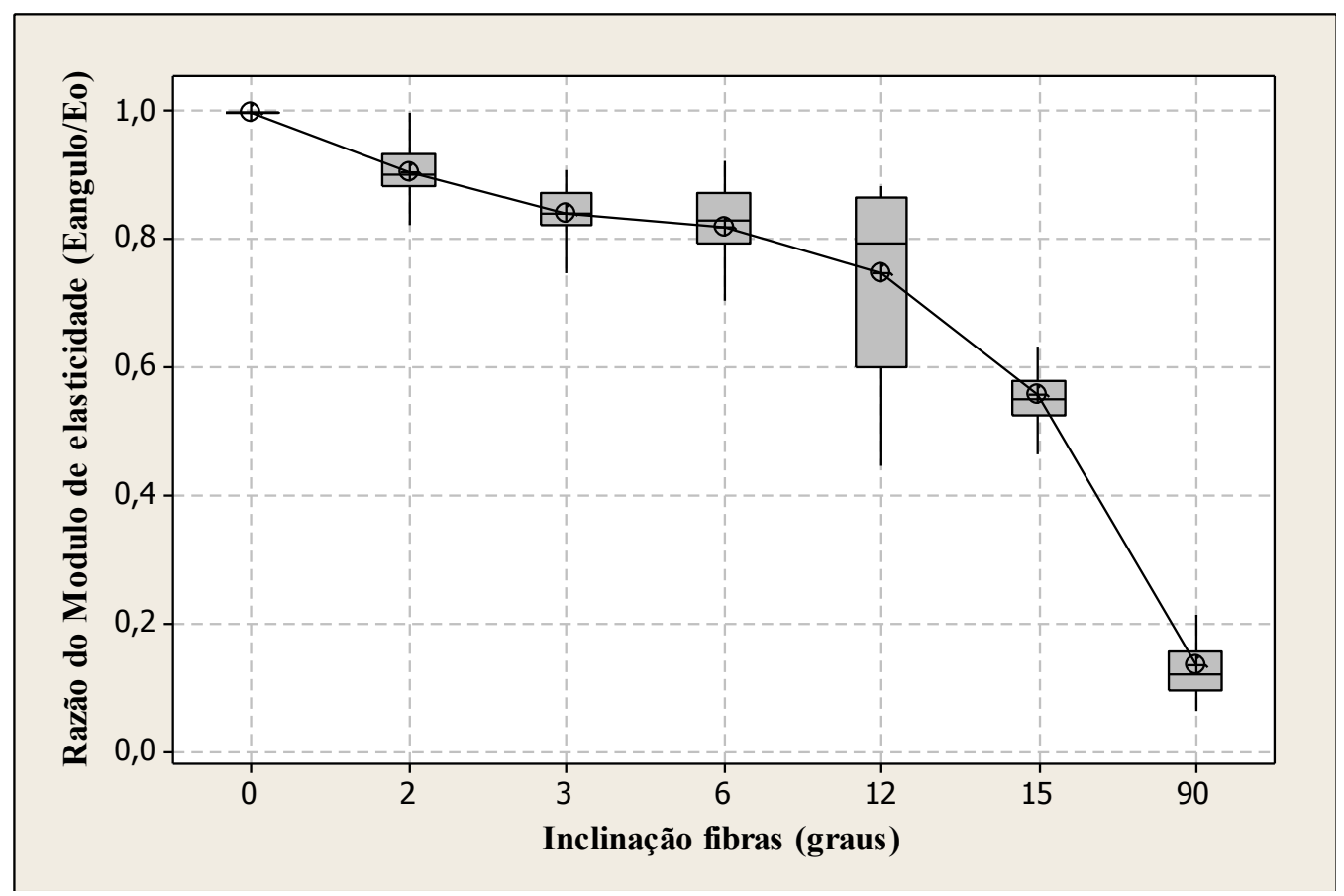

Figura 5: Resultados experimentais da razão dos módulos de elasticidade, para todas as espécies de madeira.

Na Figura 6 é apresentado o gráfico da relação do módulo de elasticidade inclinado às fibras/módulo de elasticidade paralelo às fibras $\mathrm{x}$ inclinação das fibras, dos valores experimentais (incluindo o intervalo de confiança) e das 6 equações com o expoente sugerido pelos autores das equações [13, 19, 22, 23, 24]. Notase que as curvas com todas as equações estão fora do intervalo de confiança dos resultados experimentais para, pelo menos, uma inclinação das fibras da madeira. Isso indica que os expoentes " $n$ " das equações devem ser diferentes dos sugeridos pelos autores.

Os resultados experimentais apresentaram uma distribuição normal, como mostra o teste de AndersonDarling ( $p$-value $>0,05)$. Através da análise estatística da hipótese de diferença nula, para a comparação entre pares de indivíduos e grupos de indivíduos, foram comparados os valores experimentais com os equivalentes calculados pelas equações 1 a 6 , utilizando a variação dos expoentes (n) das funções trigonométricas.

Com o objetivo de determinar qual equação representa melhor os resultados experimentais com elevada significância estatística, foram traçados gráficos obtidos a partir da análise estatística das diferenças entre os valores experimentais e as equações 1 a 6 com expoente "n" que forneceu o melhor ajuste. Nos gráficos que representam os histogramas dos resíduos da diferença, observou-se que os valores das diferenças entre os experimentais e os obtidos pelas equações da norma alemã DIN 1052, não apresentam tendência de uma distribuição normal. Já nos gráficos boxplot dos resíduos da diferença entre os valores experimentais e os que representam a equações de, Keylwerth e Tsai-Hill, observa-se que os intervalos de confiança são amplos e tem muitos pontos "outlier" com amplitudes das diferenças elevadas. Assim pode-se inferir que as equações da Hiperbólica, Karlsen e de Hankinson são as que melhor representam os resultados experimentais. 


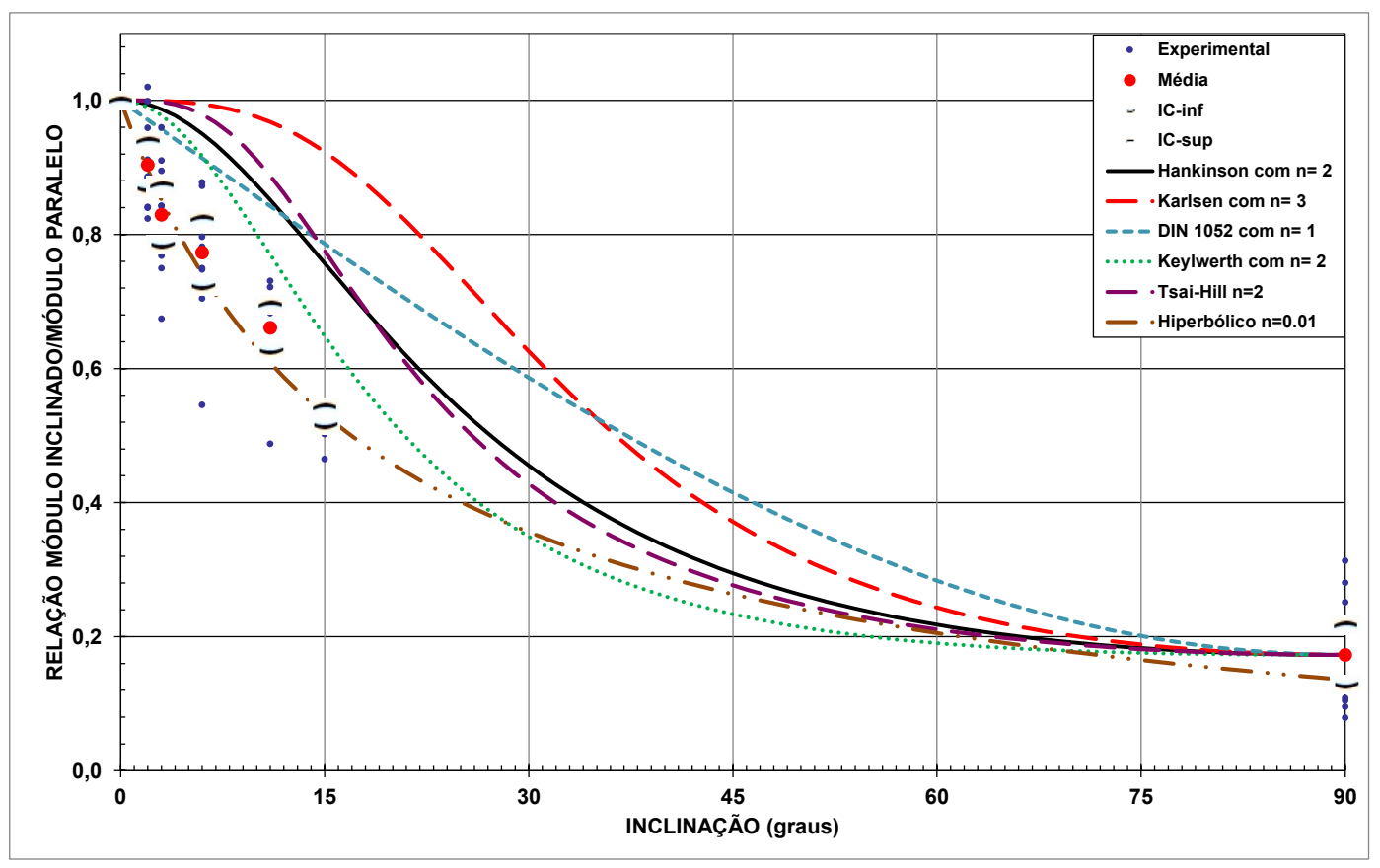

Figura 6: Relação do módulo de elasticidade inclinado às fibras/mód'ulo de elasticidade paralelo às fibras $\mathrm{x}$ inclinação das fibras, com os expoentes indicados pelos autores.

Finalmente, com a finalidade de visualizar a variação da relação dos módulos em função da inclinação das fibras, de todas as equações, dentro do intervalo de confiança das relações dos módulos experimentais (nível de significância de $95 \%$ ), se elaborou a representação gráfica das relações dos módulos experimentais e das equações, utilizando o expoente "n" que resultou em uma maior significância estatística (Figura 7). Da análise desses gráficos e levando em consideração as análises estatísticas pode-se afirmar que em ordem de significância as equações que melhor representam os valores experimentais são: hiperbólica (equação 8), Karlsen (equação 9) e Hankinson (equação 10).

$$
\begin{aligned}
& \frac{E_{d \theta}}{E_{d 0}}=\frac{\frac{E_{d 0}}{E_{d 0}} \times \frac{E_{d 90}}{E_{d 0}}}{\frac{E_{d 0}}{E_{d 0}} \sinh (0,01 . \theta)+\frac{E_{d 90}}{E_{d 0}} \cosh (0,01 . \theta)} \quad \text { ou } \quad \frac{E_{d \theta}}{E_{d 0}}=\frac{2 \frac{E_{d 0}}{E_{d 0}} \times \frac{E_{d 90}}{E_{d 0}}}{\mathrm{e}^{0,01 . \theta}\left(\frac{E_{d 0}}{E_{d 0}}+\frac{E_{d 90}}{E_{d 0}}\right)+\mathrm{e}^{-0,01 . \theta}\left(\frac{E_{d 90}}{E_{d 0}}-\frac{E_{d 0}}{E_{d 0}}\right)} \\
& \frac{E_{d \theta}}{E_{d 0}}=\frac{\frac{E_{d 0}}{E_{d 0}}}{1+\left(\frac{\frac{E_{d 0}}{E_{d 0}}}{\frac{E_{d 90}}{E_{d 0}}}+1\right) \times \sin (\theta)^{1,2}} \\
& \frac{E_{d \theta}}{E_{d 0}}=\frac{\frac{E_{d 0}}{E_{d 0}} \times \frac{E_{d 90}}{E_{d 0}}}{\frac{E_{d 0}}{E_{d 0}} \times \sin (\theta)^{1,33}+\frac{E_{d \theta}}{E_{d 0}} \times \cos (\theta)^{1,33}}
\end{aligned}
$$




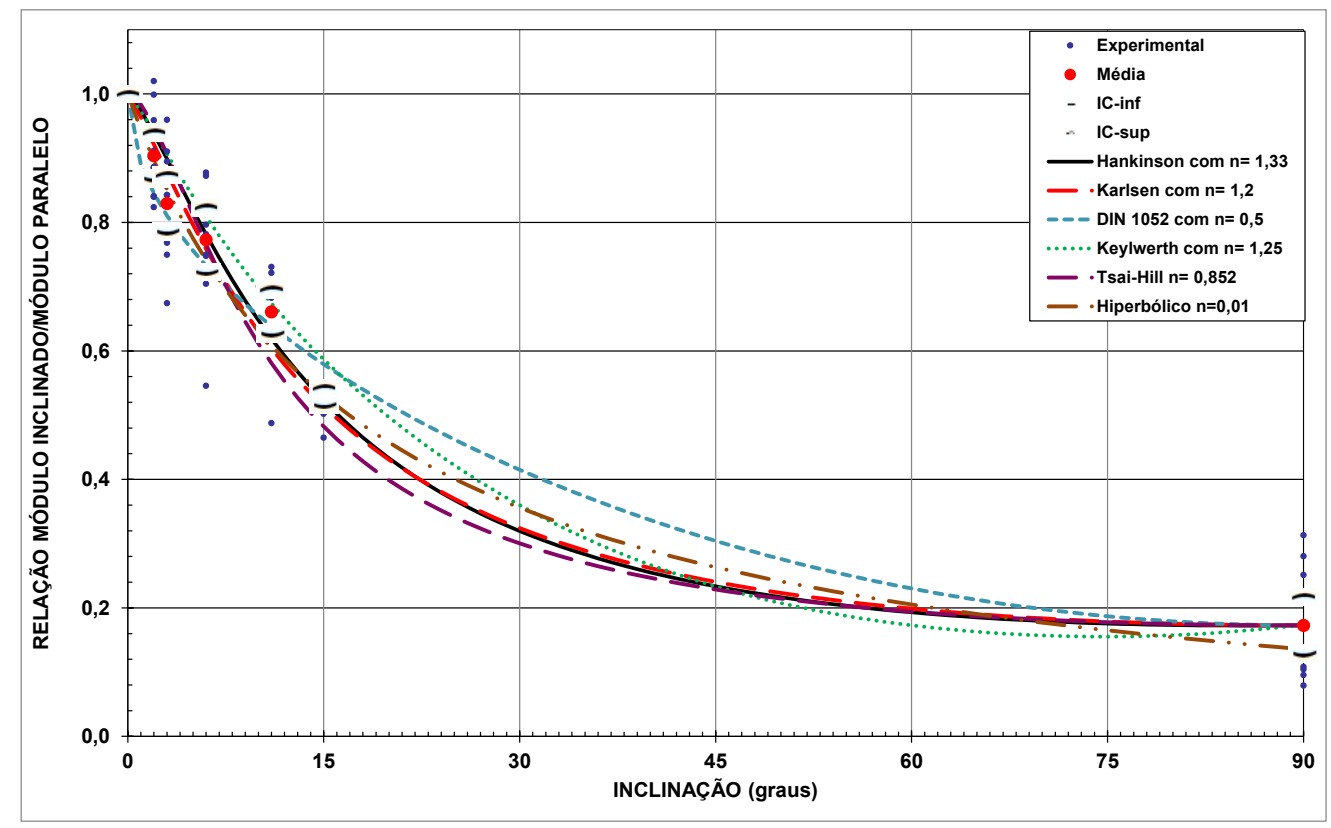

Figura 7: Relação do módulo de elasticidade inclinado às fibras/módulo de elasticidade paralelo às fibras $\mathrm{x}$ inclinação das fibras, com os expoentes " $n$ " que deu maior significância.

\section{CONCLUSÕES}

É possível utilizar o tomógrafo acústico para determinar o módulo de elasticidade inclinado às fibras, com apenas um ensaio experimental.

Todos os seis modelos matemáticos avaliados não apresentam significância estatística em seu formato original. Com as modificações feitas nos modelos os que apresentaram significância foram apenas os modelos: hiperbólica, Karlsen e Kankinson, portanto estes são os mais indicados para estimar os valores do módulo de elasticidade dinâmico em função da inclinação das fibras.

\section{AGRADECIMENTOS}

Ao Conselho Nacional de Desenvolvimento Científico e Tecnológico (CNPq) e Fundação de Apoio à Pesquisa do Estado de Minas Gerais (FAPEMIG) pelo apoio financeiro dado a esta pesquisa.

\section{BIBLIOGRAFIA}

[1] BUCUR, V. "Acoustics of wood", Berlin, Springer-Verlag, 2006.

[2] DEFLORIO, G., FINK, S., SCHWARZE, F.W.M.R. "Detection of incipient decay in tree stems with sonic tomography after wounding and fungal inoculation”, Wood Science and Tecnology, v.42, n. 2, pp. 117-132, 2008.

[3] DIKRALLAH, A., KABOUCHI, B., HAKAM, A., et al., "Study of acoustic wave propagation through the cross section of green wood", Comptes Rendus Mecanique, v. 338, n. 2, pp. 107-112, 2010.

[4] MARIÑO, R.A., FERNANDEZ, M.E., FERNANDEZ-RODRÍGUEZ, C. et al., "Detection of pith location in chestnut lumber (Castanea sativa Mill.) by means of acoustic tomography and longitudinal stresswave velocity", Eur. J. Wood Prod, v. 68, n. 2, pp. 197-206, 2010.

[5] ZHANG, H., WANG, X., SU, J. "Experimental investigation of stress wave propagation in standing trees", Holzforschung, v. 65, n. 5, pp. 743-748, 2011.

[6] LI, L., WANG, X., WANG, L., et al., "Acoustic tomography in relation to 2D ultrasonic velocity and hardness mappings", Wood Sci Technol, v. 46, n. 1-3, pp. 551-561, 2012.

[7] CHIMENTI, D. E. "Review of air-coupled ultrasonic materials characterization", Ultrasonics, v. 54, n. 7, pp. 1804-1816, 2014.

[8] GILBERT, E.A., SMILEY, E. T., "PiCUS Sonic Tomography for the quantification of decay in white oak (Quercus Alba) and hickory (Carya spp.)", J Arboric, v. 30, n. 5, pp. 277-280, 2014. 
[9] WANG, X., WIEDENBECK, J., LIANG, S. "Acoustic tomography for decay detection in black cherry trees," Wood Fiber Sci, v. 41, n. 2, pp. 127-137, 2009.

[10] AMODEI, J.B., OLIVEIRA, B.R.U., GURGEL, M.M., et al., "Avaliação Preliminar da Qualidade da Madeira de Tectona grandis L. f. através da Tomografia de Impulso", Floresta e Ambiente, v. 17, n. 2, pp. 124-128, 2010.

[11] LI, G., WANG, X., FENG, H., et al., "Analysis of wave velocity patterns in black cherry trees and its effect on internal decay detection", Computers and Electronics in Agriculture, v. 104, pp. 32-39, 2014.

[12] ALVES, R.C., MANTILLA, J.N.R, BREMER, C.F., et al., "Application of Acoustic Tomography and Ultrasonic Waves to Estimate Stiffness Constants of Muiracatiara Brazilian Wood", Bioresources, v. 10, n. 1, pp. 1845-1856, 2015.

[13] KOLLMANN, F.F.P., CÔTÉ, W.A. Principles of Wood Science and Technology: I. Solid Wood, Springer, Berlin, 1968.

[14] BODIG, J., JAYNE, B.A. Mechanics of Wood and Wood Composites, Van Nostrand Reinhold, New York, 1982.

[15] DINWOODIE, J.M. "Timber: A review of the structure-mechanical property relationship", Journal of Microscopy, v. 104, n.1, pp. 3-32, 1975.

[16] CARRASCO, E.V.M., MANTILLA, J.N.R. "Applying failure criteria to shear strength evaluation of bonded joints according to grain slope under compressive load", International Journal of Engineering \& Technology, v. 13, n. 4, pp. 19-25, 2013.

[17] CARRASCO, E.V.M., MANTILLA, J.N.R., "Failure criteria for shear strength evaluation of bonded joints according to grain slope under tension load", BioResources, v. 10, n. 2, pp. 3602-3614, 2015.

[18] CARRASCO, E.V.M., MANTILLA, J.N.R. "Influência da inclinação das fibras da madeira na sua resistência ao cisalhamento", Ciência Florestal, v. 26, n. 2, pp. 535-543, 2016.

[19] WOODWARD, C., MINOR, J. "Failure theories for Douglas-fir in tension", Journal of Structural Engineering, v 114, n.12, pp. 2808-2813, 1988.

[20] OH, S.C., "Applying failure criteria to the strength evaluation of 3-ply laminated veneer lumber according to grain direction by uniaxial tension test", Construction and Building Materials, v. 25, n.3, pp. 14801484, 2011.

[21] ANÔNIMO (1979), NBR 7190: Projeto de estruturas de madeira. Rio de Janeiro, ABNT - Associação Brasileira de Normas Técnicas.

[22] KARLSEN, G.G., BOLSHAKOV, V.V., KAGAN, M.Y., et al., Wooden Structures, Mir Publishers, Moscow, 1967.

[23] ANÔNIMO (2008), DIN 1052: Design of Timber Structures - General Rules and Rules for Buildings. Berlin, DIN - Deutsches Institut Für Normung.

[24] WOODWARD, C., The elastic and strength properties of Douglas fir in the radial-longitudinal plane, Ph.D. Dissertation, New Mexico State University, Las Cruces, NM., 1986. 